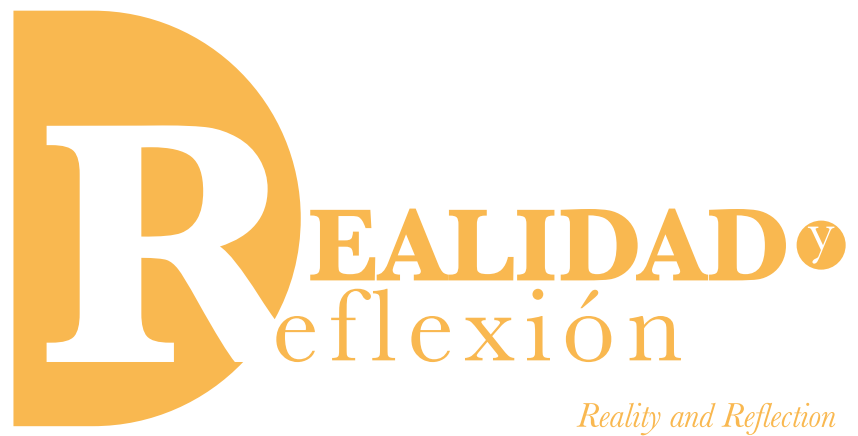

ISSN 1992-6510

Año 18, N 48, San Salvador, El Salvador, Centroamérica. Revista Semestral Julio-Diciembre 2018

YEAR 18, N 48, SAN SALVAdor, EL SALVADOR, CENTRAL AMERICA. SEMESTRAL JOURNAL JuLY-DECEMBER 2018

\title{
¿Twitter herramienta de campaña? Una mirada a la red social el día de las elecciones para diputaciones y concejos municipales 2018 en E1 Salvador
}

\author{
Twitter campaign tool? A look at the twitter social \\ network on the day of the elections for deputies and \\ mayors 2018 in El Salvador
}

\author{
Ana Evangelina Aguilar \\ Licenciada en Periodismo y Comunicaciones \\ Universidad Centroamericana José Simeón Cañas, UCA \\ Máster en Asesoría de Imagen y Consultoría Política \\ Universidad de Salamanca, España. \\ Investigadora para el área de Comunicaciones ICTI-UFG \\ aeaguilar@ufg.edu.sv \\ Fecha de recepción: 7 de septiembre de 2018 \\ Fecha aprobación: 3 de diciembre de 2018 \\ DOI:
}

\section{RESUMEN}

Este artículo tiene como objetivo agregar elementos al debate latinoamericano sobre el uso de las redes sociales en los procesos electorales. Se plantea una discusión inicial sobre el alcance de la comunicación política y las redes sociales advirtiendo sobre el poco valor que los candidatos y partidos políticos salvadoreños le atribuyen a Twitter en un día tan importante en el desarrollo de la campaña electoral. El uso, informativo y comunicacional, de las redes sociales debería de ser clave en el día de elecciones, pero ni los candidatos, ni los partidos políticos tienen clara su utilización.

Palabras clave: Twitter, redes sociales, comunicación política, campañas electorales, E1 Salvador, 2018.

ABSTRACT

The objetive of this article is to provide elements for the Latin American debate about the use of social media in the electoral processes. The article puts forward an initial discussion about the scope of political communication and social media, warning about the low value that Salvadoran candidates and political parties attribute to Twitter on such an important day in the development of the electoral campaign. The informative and communicational usage of social media should be key during the election day, but nor candidates nor political parties have clarity in the funtions of such network.

Keywords: Social media, Twitter, political communication, electoral campaign, El Salvador, 2018. 


\section{Introducción}

La comunicación política desarrolla un papel central en todo proceso político, de hecho, la mayor parte de la actividad comunicacional se centra en el desarrollo de la campaña electoral. Los partidos políticos tienen una cuidadosa atención al diseño de los mensajes y a las formas cómo presentan el discurso, tienden a diversificar los canales y las herramientas por los cuales se comunican con sus electores.

Uno de los precursores en América de esta diversificación de herramientas fue Barack Obama quien para el evento electoral del 2008 hizo uso de una campaña con un importante papel de las redes sociales y del internet, dejando a la televisión de lado como medio hegemónico (Mancera \& Pano, 2013).

Así los partidos políticos y los candidatos han encontrado en las redes sociales un espacio democrático de comunicación y en donde el costo publicitario de la campaña puede ser menor al de los medios de comunicación tradicionales. Las redes sociales también pueden hacerle creer al elector que tiene la posibilidad de acercarse a su candidato o partido debido a la potencialidad de interacción que tienen las redes, de hecho éstas son más atractivas en latitudes en donde los candidatos tienden a individualizar su campaña. Los partidos o candidatos tienen la posibilidad de diseñar mensajes o discursos bien específicos, tanto que pueden crear una relación con el elector. La finalidad es acercar a los candidatos y electores.

Las posibilidades de interacción y proximidad que permiten las redes sociales han contribuido a su consolidación en la sociedad como una alternativa. De hecho Javier Echeverría plantea un nuevo modelo de espacio social que denomina "tercer entorno" el cual se coloca por encima de los dos ambientes ya existentes, el entorno rural -el cual tiene que ver con el campo-y el entorno de la ciudad -que tiene que ver con los sectores urbanos-. Echeverría sugiere que un tercer entorno no invalidará a los otros dos sino que se presenta en coexistencia con estos. Así una sociedad puede tener una plasmación urbana como rural o electrónica (Echeverría, 1999).

Por tanto, dicho entorno genera oportunidades de construir perfiles públicos, crear nuevos vínculos que en muchas ocasiones de manera offine serían muy difíciles y costosas de construir, convirtiendo a las propuestas online en parte de un espacio ideal para la difusión de ideas y propuestas políticas.

Entonces, en un mundo tan interconectado resulta difícil concebir campañas electorales que no contemplen el uso planificado de los recursos que internet ofrece. Investigaciones en el campo de la comunicación, la comunicación política, la sociología y la antropología están permitiendo que se conozca el papel de las redes sociales en distintos periodos, tanto electorales como fuera de este campo; pero muchos de ellos señalan que es en el periodo electoral cuando se ve intensificado el uso de dichos perfiles.

Con una cifra muy por encima de los 300 millones de usuarios en el mundo, el servicio de microblogging llamado Twitter se ha mantenido como el canal de comunicación preferido por los líderes mundiales y los Gobiernos. Lo usan Jefes de Estado y los Gobiernos de más de 
178 países; tanto como el $92 \%$ de los Estados miembros de Naciones Unidas, de acuerdo a Triplomacy, el estudio anual que realiza Burson-Marsteller sobre el uso que hacen los líderes mundiales de los medios digitales (Tecnología hecha palabra, 2017).

A pesar de la importancia que tienen las redes sociales y el Twitter en el mundo político, en un país como El Salvador el uso y efecto de las redes sociales no está muy estudiado. El presente trabajo pretende contribuir al conocimiento, que poco a poco se va acumulando a nivel nacional, sobre campañas electorales y redes sociales mediante una investigación empírica que se centra en Twitter y que emplea el estudio de caso como metodología; para ello se concentra la atención en el impacto de Twitter el día de las elecciones para diputaciones y concejos municipales 2018.

Más allá de constatar si los partidos políticos y algunos candidatos tienen presencia o no en Twitter, se pretende indagar si los funcionarios utilizan las potencialidades de esta red social o si, por el contrario, su interés se limita a una simple cuestión de moda.

\section{Objetivos y metodología}

El objeto de estudio de esta investigación es la actividad digital que mantuvieron en sus perfiles de Twitter los partidos políticos que participaron en la contienda electoral, los secretarios generales de dichos partidos políticos y algunas cuentas de políticos durante el 4 de marzo de 2018, día que se desarrollaron las elecciones para diputaciones y concejos municipales 2018 de El Salvador. Además de las cuentas de Twitter también se realizó un seguimiento de las palabras, hashtags y cuentas que más presencia tuvieron durante el día de elecciones.

La metodología empleada en esta investigación corresponde a la técnica del análisis de contenido cuantitativo, sugerido en el modelo de análisis de contenido propuesto por Andréu-Abela (2012) en donde los procesos de comprensión y conceptualización de los datos mediante los procedimientos de fragmentación y etiquetado de la información como paso previo al proceso de codificación determinan las interacciones de los actores -tácticas y estrategias-, y consecuencias de los fenómenos sociales, es decir, análisis de las estructuras en procesos de cambio e interacción social.

Además, a partir de una selección de variables cualitativas se realizaron capturas de pantalla de las distintas cuentas de Twitter con las que se elaboró un diario de campo del día 4 de marzo de 2018. Las herramientas que se usaron para el seguimiento de tendencias de los distintos perfiles fueron Twitonomy y Trendsmap. Por un lado, Twitonomy es una aplicación web que permite analizar una cuenta de Twitter por medio de diferentes métodos. Este servicio permite generar analíticas visuales y detalladas de tweets, retweets, respuestas, menciones y hashtags. Por otro lado, Trendsmap es una herramienta para estar al tanto, en tiempo real, de qué es lo que está sucediendo en una determinada región, es decir cuáles son los hashtags más populares y sobre qué tendencias se está hablando en Twitter, pero Trendsmap no es un instrumento de tendencias cualquiera, lo caracteriza su tecnología de GeoIP que gracias a Google, permite tener cierta 
precisión sobre la ubicación de un hashtag en una determinada región y hora.

El objetivo de la investigación es conocer el tipo de tweets, es decir qué tipo de interacción, tuvieron los perfiles seleccionados el día de las elecciones para diputaciones y concejos municipales 2018. Los temas que dominaron sus perfiles así como las palabras, los usuarios y los hashtags con más interacción entre las siete de la mañana y las 10 de la noche del día de elecciones.

Los perfiles que se escogieron para el análisis de su actividad en Twitter son los de los secretarios generales de los principales partidos en contienda y que tienen cuenta en la red social de Twitter, así como los candidatos de tres de los departamentos con más cúmulo de twitteros en el país que son San Salvador, Santa Ana y San Miguel, de igual forma se tomaron los candidatos de los municipios de Santa Tecla y Antiguo Cuscatlán municipios del departamento de La Libertad. También se analizaron las cuentas del Alcalde de la ciudad capital de San Salvador y del diputado presidente de la Asamblea Legislativa.

Es importante señalar que este estudio se limita a los perfiles de algunos candidatos municipales de los principales partidos en contienda y no de los candidatos a diputado ya que el número de candidatos era bastante significativo. También es revelador el hecho de que, al momento de hacer esta investigación, secretarios generales de algunos partidos políticos como el FMLN, GANA, PSD y FPS no tienen perfiles en Twitter y que el perfil del candidato a alcalde de Santa Tecla por el partido FMLN, Vicente Coto, era privado por lo que no se pudo analizar.

Tabla n. ${ }^{\circ} 1$

Cuentas de twitter analizadas

\begin{tabular}{|l|l|l|}
\hline \multicolumn{3}{|c|}{ Candidatos para alcaldías municipales } \\
\hline Ernesto Muyshondt & $@$ emuyshondt & Candidato a Alcalde de San Salvador ARENA \\
\hline Jackeline Rivera & $@$ JackelineRA__ & Candidata a Alcaldesa de San Salvador FMLN \\
\hline Roberto d'Aubuisson & $@$ @rdaubuisson & Candidato a Alcalde de Santa Tecla ARENA \\
\hline Vicente Coto & $@$ CotoAlcalde & Candidato a Alcalde de Santa Tecla FMLN \\
\hline Milagro Navas & $@$ milagro_nnavas & Candidata a Alcalde de Antiguo Cuscatlán ARENA \\
\hline Luis Rodríguez & $@$ @Rodriguez_SV & Candidato a Alcalde de Antiguo Cuscatlán FMLN-CD \\
\hline Milena de Escalón & $@$ Milena_Escalon & Candidata a Alcalde de Santa Ana ARENA \\
\hline Cecibel Lau & $@$ cecibel_lau & Candidata a Alcalde de Santa Ana FMLN \\
\hline Miguel Pereira & $@$ Miguelpereirasv & Candidato a Alcalde de San Miguel FMLN \\
\hline Will Salgado & $@$ WILLSALGADO & Candidato a Alcalde de San Miguel GANA \\
\hline & \multicolumn{2}{|c|}{ Secretarios de partidos políticos } \\
\hline Mauricio Interiano & $@$ minteri & Secretario Alianza Republicana Nacionalista ARENA \\
\hline Medardo González & ----------- & Secretario Frente Farabundo Martí FMLN \\
\hline Manuel Rodríguez & $@$ memourgente1 & Secretario Partido de Conciliación Nacional PCN \\
\hline Andrés Rovira & ------------ & Secretario Gran Alianza GANA \\
\hline
\end{tabular}




\begin{tabular}{|c|c|c|}
\hline Rodolfo Parker & @rodolfo_parker & Secretario Partido Demócrata Cristiano PDC \\
\hline Juan José Martel & $@$ @jmartel & Secretario Cambio Democrático CD \\
\hline Jorge Meléndez & -------------- & Secretario Partido Social Demócrata PSD \\
\hline Oscar Lemus & ---------------- & Secretario Fraternidad Patriota Salvadoreña FPS \\
\hline \multicolumn{3}{|c|}{ Partidos Políticos } \\
\hline ARENA & @ARENAOFICIAL & \\
\hline FMLN & @FMLNoficial & \\
\hline $\mathrm{PCN}$ & $@$ @PCN_Oficial & \\
\hline GANA & @GANAOFICIAL & \\
\hline PDC & $@$ PDC_OFICIAL & \\
\hline $\mathrm{CD}$ & $@$ CD_ESA & \\
\hline PSD & @PSD_OFICIAL_SV & \\
\hline FPS & 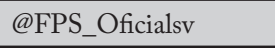 & \\
\hline \multicolumn{3}{|c|}{ Otros políticos } \\
\hline Guillermo Gallegos & $@$ GGallegos24 & Presidente diputado Asamblea Legislativa. \\
\hline Nayib Bukele & $@$ nayibbukele & Alcalde de San Salvador. \\
\hline
\end{tabular}

Fuente: elaboración propia.

Así, en el día seleccionado para el análisis y con la intención de monitorizar la actividad de cada perfil se aprovechó la plataforma de TweetDeck, herramienta oficial creada por Twitter para gestionar y controlar varias cuentas desde un solo escritorio o panel de control, con la que se creó una lista de seguimiento compuesta por 25 cuentas de las que se recopilaron los tweets publicados el día 4 de marzo de 2018. Con dichos datos se elaboró una ficha de análisis aplicada en este estudio que incluyó distintas categorías como denuncia, propuestas, actividades, agradecimiento, llamado al voto y mensajes.

\section{Ver Figura n. ${ }^{\circ} 1$.}

Cada una de las variables está relacionada con la actividad que desarrollaron los candidatos y partidos políticos durante su campaña electoral; pero que puede verse incrementada o disminuida el día principal de la votación.

Por otro lado, se monitoreó, con la herramienta Trensmap, cada dos horas desde las ocho de la mañana a las diez de la noche, a las palabras más compartidas en el país, así como los hashtags más utilizados y los usuarios que tuvieron mayor interacción. Con este mapa de elementos se podrá observar cómo fueron cambiando las conversaciones en la red social de Twitter el día de las elecciones, así como los usuarios de la red de microblogging con mayor interacción.

\section{Resultados}

Para esta investigación, como se menciona en la metodología, se analizaron 25 cuentas de la red de Twitter. En ellas se dieron seguimiento 
Luis Rodriguez@LRodriguez_SV.4 mar.

Hoy llegó el momento, pase lo que pase, ya hicimos ganar a \#AntiguoCuscatlán, porque les demostramos que esta ciudad es un diamante en bruto con posibilidad de ser 100 veces mejor.

Aquí comienza el futuro!

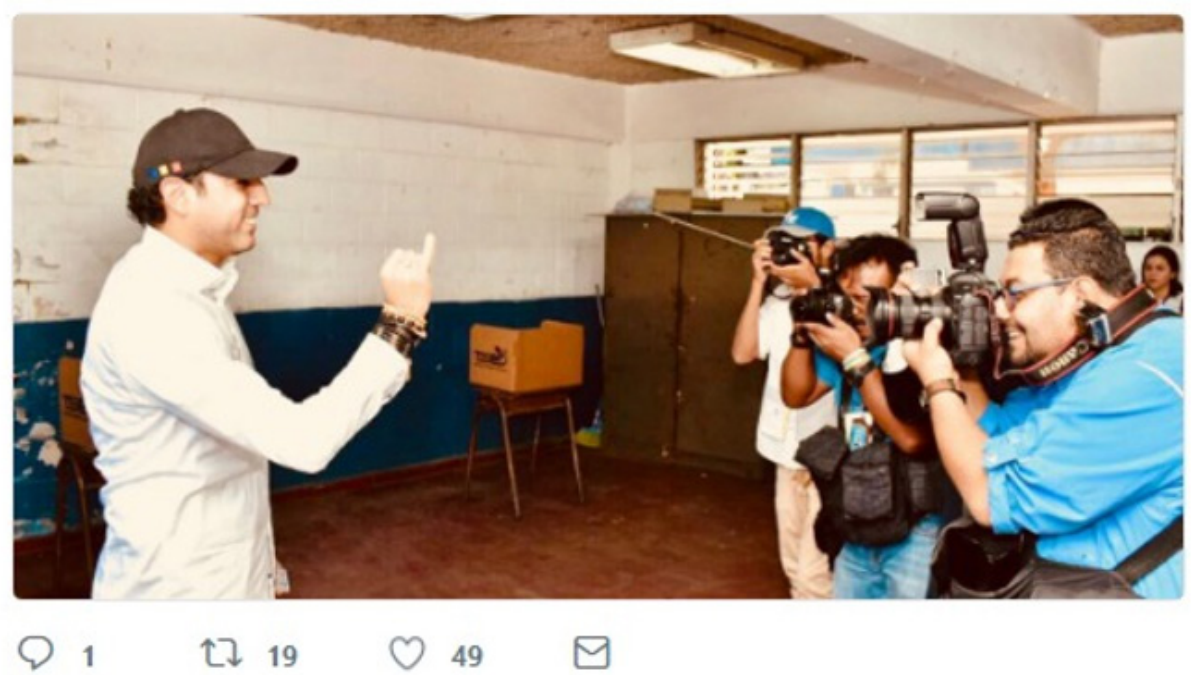

Figura n. ${ }^{1}$. Categoría "mensaje". Fuente: Twitter de Luis Rodríguez [@LRodriguez_SV]

a cada una de las publicaciones realizadas y se agruparon en las siguientes categorías:

- Denuncia: los partidos políticos o candidatos podían hacer comentarios de las anomalías o situaciones fuera de la ley de la jornada electoral.

- Propuestas: los candidatos o partidos podrían colocar mensajes de sus planes de gobierno municipal, así como de sus plataformas legislativas.

- Actividades: en esta variable se clasificaron los indicadores de presencia de los candidatos, secretarios generales de partido o también información sobre las distintas acciones que estarían realizando.

- Agradecimiento: las muestras de reconocimiento y gratitud que los candidatos o partidos hicieron a su militancia o al pueblo salvadoreño.

- Llamar a votar: El Salvador es un país en donde el abstencionismo en las urnas, en las últimas elecciones, ha llegado casi al $50 \%$ de la población, por lo que es común que los candidatos y partidos políticos desarrollen 
su papel de incentivo al voto utilizando propuestas o frases inspiradoras para que sus seguidores salgan a votar.

- Mensajes: a diferencia de las propuestas de campaña, los mensajes pueden ser frases o menciones políticas de los candidatos hacia sus seguidores, así como frases que se mencionaron en las visitas de los candidatos o secretarios generales de los partidos en los medios de comunicación.
Así, el día de las votaciones, los tweets con mensajes hacia la población (320 tweets), las menciones sobre las actividades que estaban realizando los candidatos o partidos (258 tweets) y los llamados a votar fueron las tres primeras categorías de mensajes que los investigados enviaron a sus seguidores. Le siguen con 53 tweets las propuestas de campaña, los mensajes de agradecimiento (34 tweets) y las denuncias (24 tweets) entre las que se encontraba el retraso en la apertura de centros de votación y la situación en CIFCO con los cortes de energía eléctrica.

\section{Gráfico n. ${ }^{\circ} 1$}

Categorización de tweets

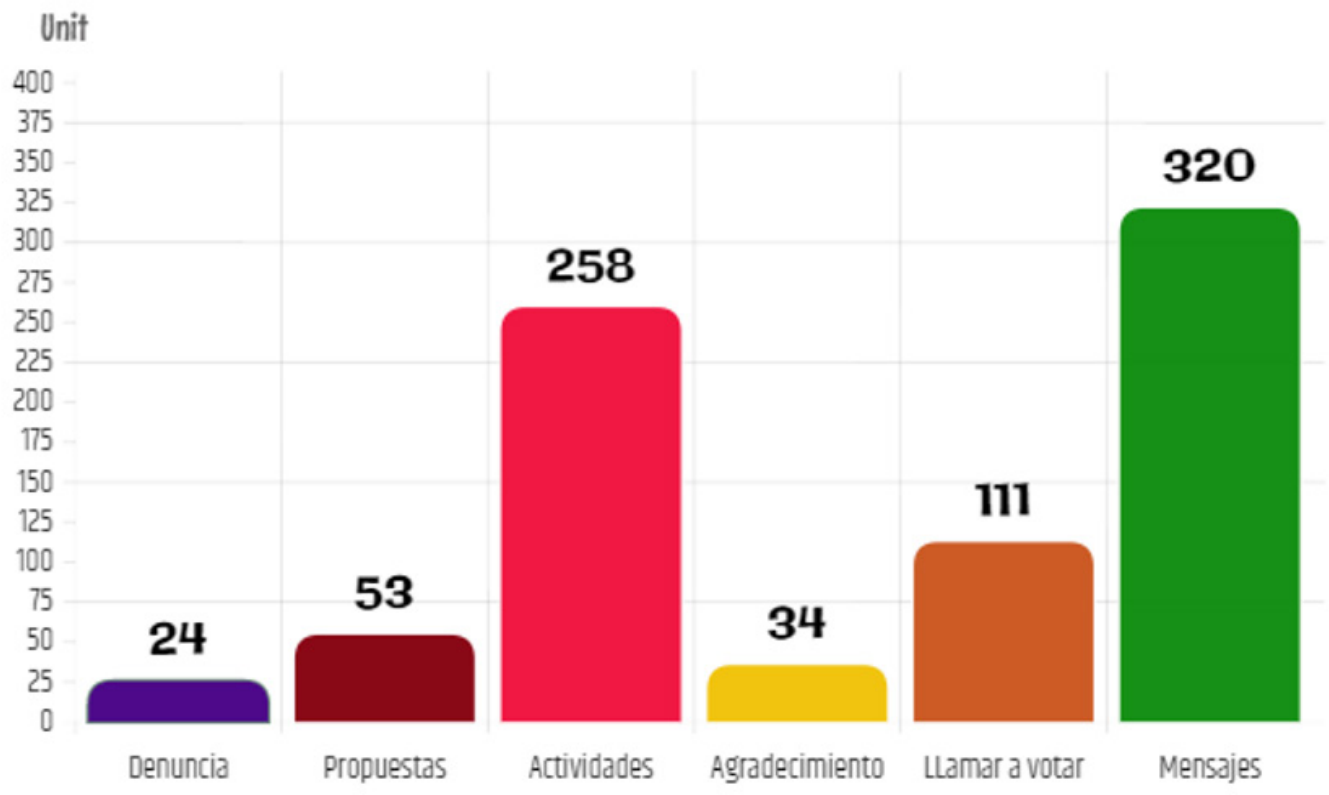




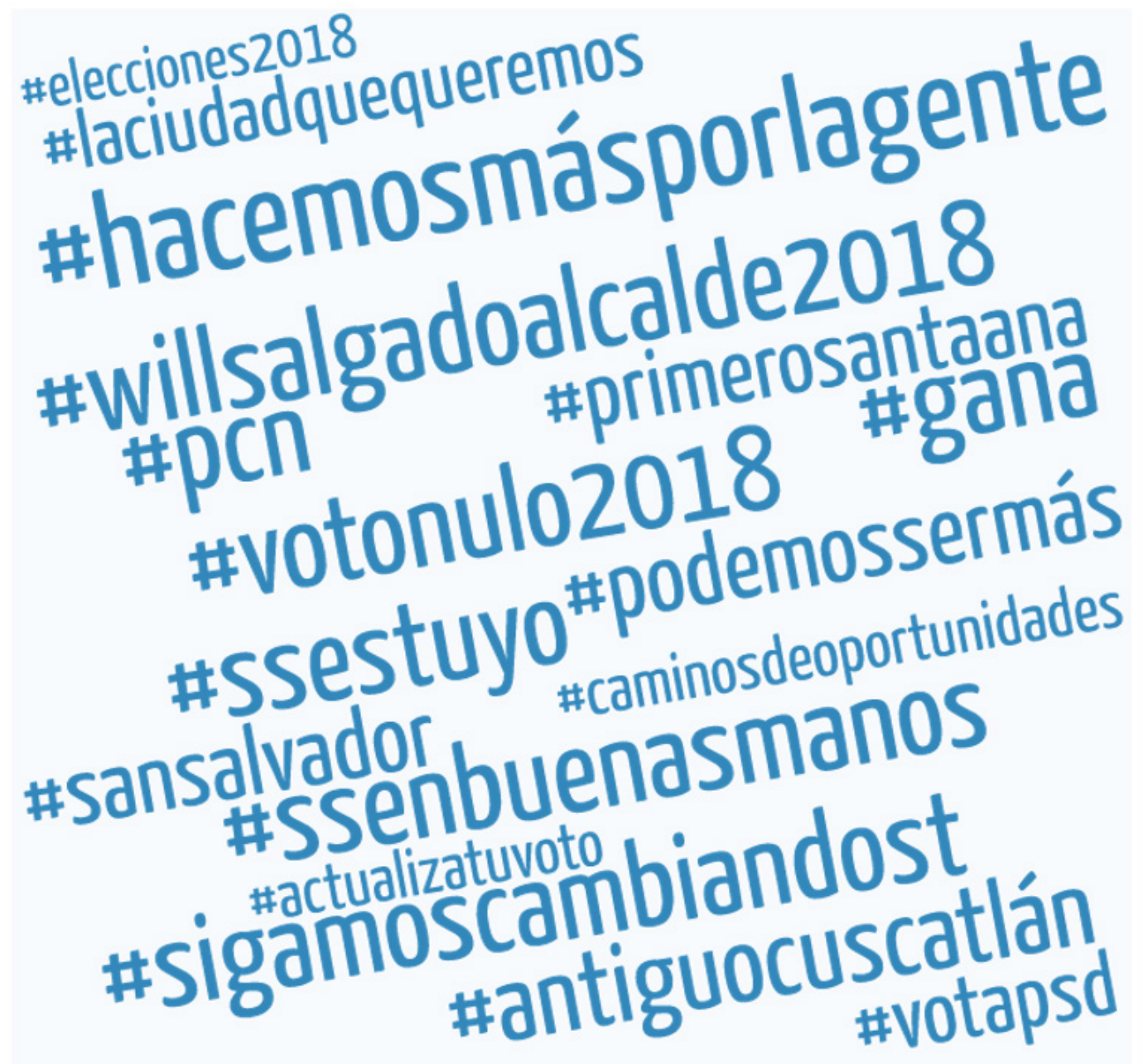

Figura . $^{\circ}$ 2. Hashtags utilizados por las cuentas investigadas.

De forma general, también se comprobó cuáles fueron los hashtags que cada una de las cuentas utilizó el día de las elecciones, coincidiendo que quienes hicieron uso de dicho recurso lo hicieron para reforzar sus eslóganes o lemas de campaña.

En donde los tres más compartidos entre los perfiles investigados fueron: \#SSEsTuyo (eslogan de campaña del candidato a alcalde de San Salvador por el partido ARENA) \#hacemosmásporlagente (eslogan de la campaña legislativa del partido FMLN) y \#SSenbuenasmanos (eslogan de campaña de la candidata por San Salvador del partido FMLN).

Del total de perfiles analizados el más activo 
fue el del partido FMLN (@FMLNOficial) con 216 publicaciones, le sigue el presidente de la Asamblea Legislativa y también candidato a la reelección Guillermo Gallegos (@GGallegos24) con 115 tweets y en tercer lugar, el entonces alcalde de San Salvador, Nayib Bukele, (@ nayibbukele) con 87 publicaciones. Por el contrario los menos activos fueron los perfiles de los partidos Social Demócrata y GANA quienes no realizaron ninguna publicación, así como el Secretario General del PCN, Manuel Rodríguez, que tuvieron cero publicaciones el día de la elección.

\section{Gráfica n. ${ }^{\circ} 2$}

Perfiles más activos en Twitter el día de la elección publicación fue directa para sus seguidores. Por otro lado, en la cuenta del partido Fraternidad Patriota Salvadoreña (@FPS_Oficialsv) se observó que sus 57 tweets fueron programados, de hecho se pudo identificar que eran tweets genéricos que también habían sido publicados en días anteriores. En ambos casos se puede observar cómo la herramienta es subutilizada y limitada.

Asimismo se pudo registrar que hubo dos perfiles, de los 25, que sí respondieron e intercambiaron tweets con sus seguidores. El primero fue el del Secretario General del Partido Cambio Democrático, quien a través de

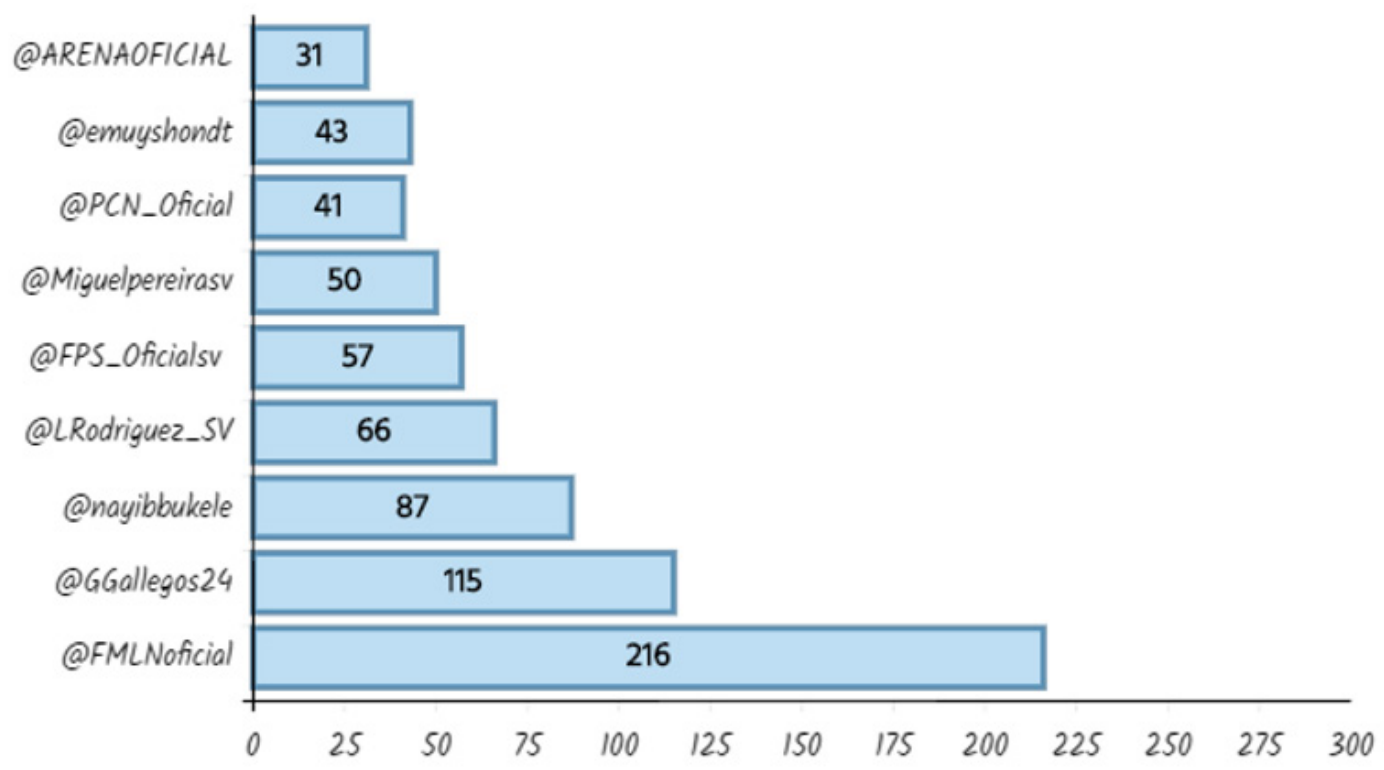

Es también importante señalar que durante el seguimiento realizado, se pudo identificar que en la cuenta del Secretario General del Partido Demócrata Cristiano, Rodolfo Parker, cada una de sus 18 publicaciones fueron retuits, ninguna su cuenta dio respuesta a dudas y comentarios de sus seguidores; y el segundo, fue el de Luis Rodríguez, candidato a la Alcaldía de Antiguo Cuscatlán que también mantuvo contacto son sus seguidores. 


\section{Los candidatos}

Los candidatos a alcalde que más interacción tuvieron en su red de Twitter fueron Luis Rodríguez, candidato a alcalde de Antiguo Cuscatlán del partido FMLN; seguido por Miguel Pereira, candidato a alcalde de San Miguel del partido FMLN, y en tercer lugar, el candidato a alcalde de San Salvador por el partido ARENA, Ernesto Muyshondt. Los tres candidatos son jóvenes y durante su campaña electoral fueron también muy activos en el uso de la red de microblogging.
Cabe mencionar que de los tres candidatos, fue Luis Rodríguez, de Antiguo Cuscatlán, quien logró tener interacción sus seguidores; es decir, que había respuestas entre sus tweets.

\section{Los secretarios generales de los partidos políticos}

Ocho fueron los partidos políticos que participaron en la contienda electoral del 4 de marzo de 2018. Los mayoritarios: ARENA y FMLN. Los partidos con más tradición electoral: PCN y PDC. Y los más recientes:

\section{Gráfico n. ${ }^{\circ} 3$}

Actividad en tweets de los candidatos a alcalde de los municipios con más tuiteros en El Salvador

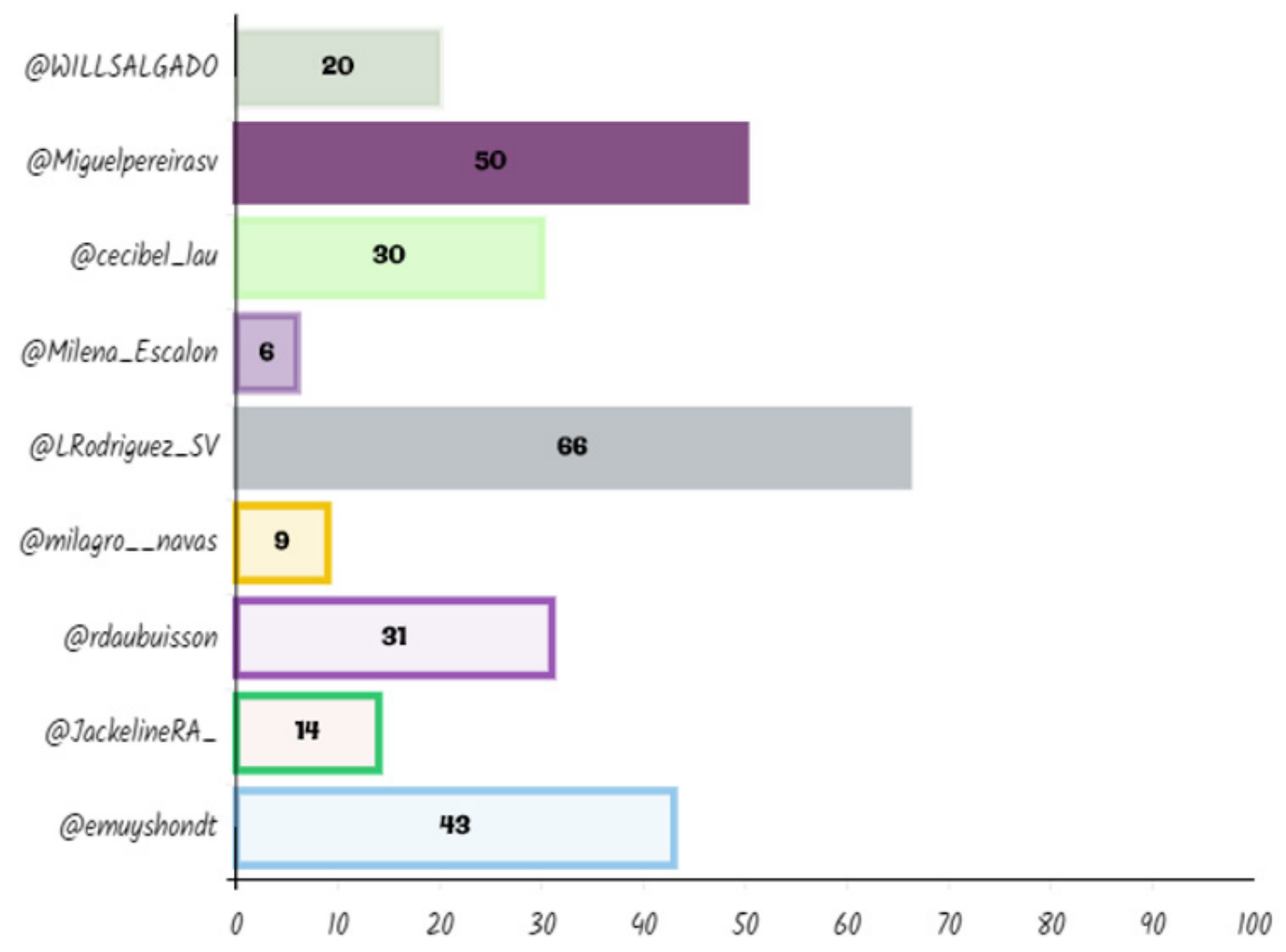


CD, GANA, PSD y FPS. Los líderes de dichos partidos son quienes inspiran a sus militantes y simpatizantes, generan opinión y tienden a forjar a sus cuadros dentro de las corrientes ideológicas. Una herramienta de comunicación con sus seguidores puede ser Twitter; pero de los ocho partidos sólo la mitad cuenta con un perfil en dicha red, por lo que se limita la interacción con sus

\section{Los partidos políticos}

E1 partido político que mayor uso realizó de la herramienta de Twitter el día de las elecciones para diputaciones y concejos municipales 2018 fue el partido oficial FMLN con una cantidad de 216 tweets. En su mayoría fueron publicaciones sobre las actividades que estaban realizando; en

\section{Gráfico n. ${ }^{\circ} 4$}

Tweets de los secretarios generales de los partidos políticos en el día de la elección

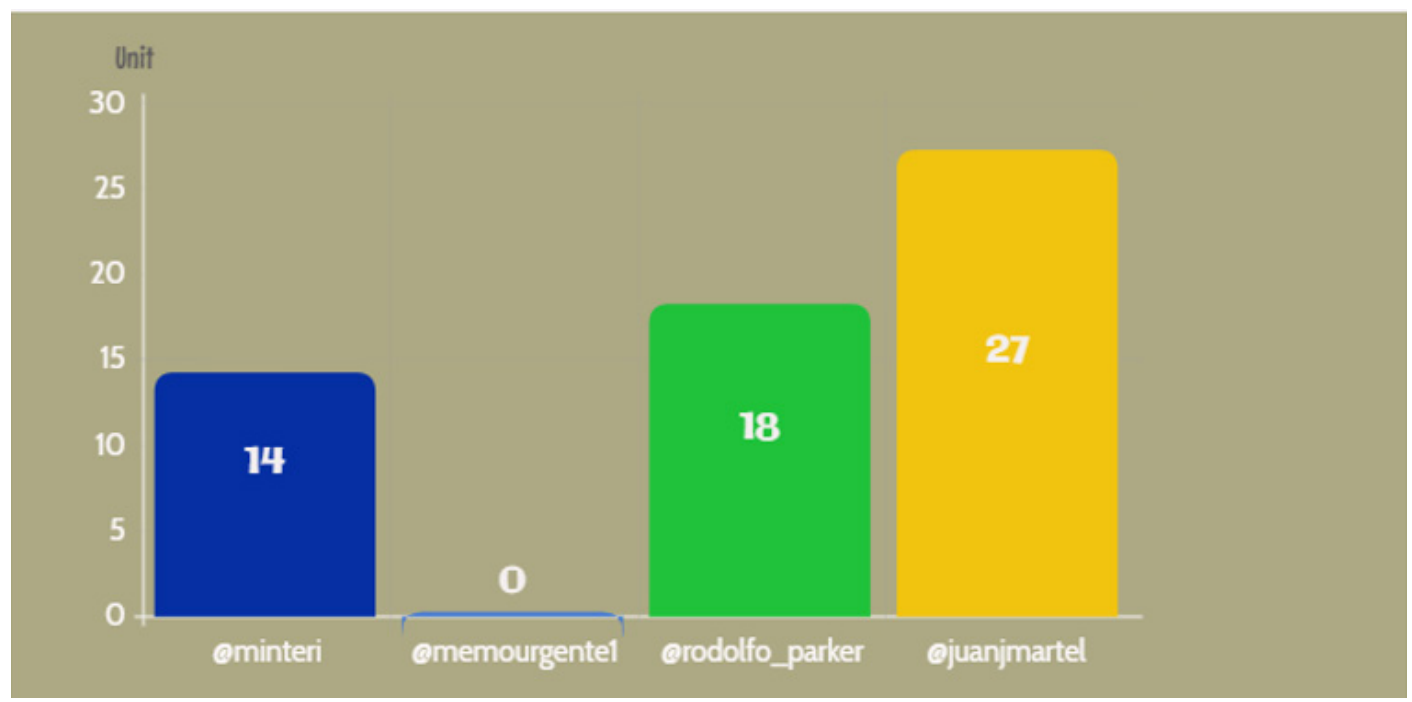

Fuente: elaboración propia.

seguidores. De los cuatro (ARENA, PCN, PDC y CD) dos de ellos, el PCN y PDC, tienen nula interacción con sus seguidores, de hecho la cuenta de Twitter del Secretario General del PCN, Manuel Rodríguez, no tuvo ninguna actividad el día de la investigación. Por otra parte, el Secretario General del PDC, Rodolfo Parker, se limitó a dar retweet de cuentas. segundo lugar, emitieron una buena cantidad de mensajes inspiradores para sus seguidores $(90,807)$ y por último tuvieron publicaciones en las que se pedía a sus seguidores que salieran a votar.

El segundo partido con un mayor número de publicaciones fue Fraternidad Patriota Salvadoreña (@FPS_Oficialsv); pero se 


\section{Gráfico n. ${ }^{\circ}$.}

Tweets publicados por los partidos políticos el día de la elección

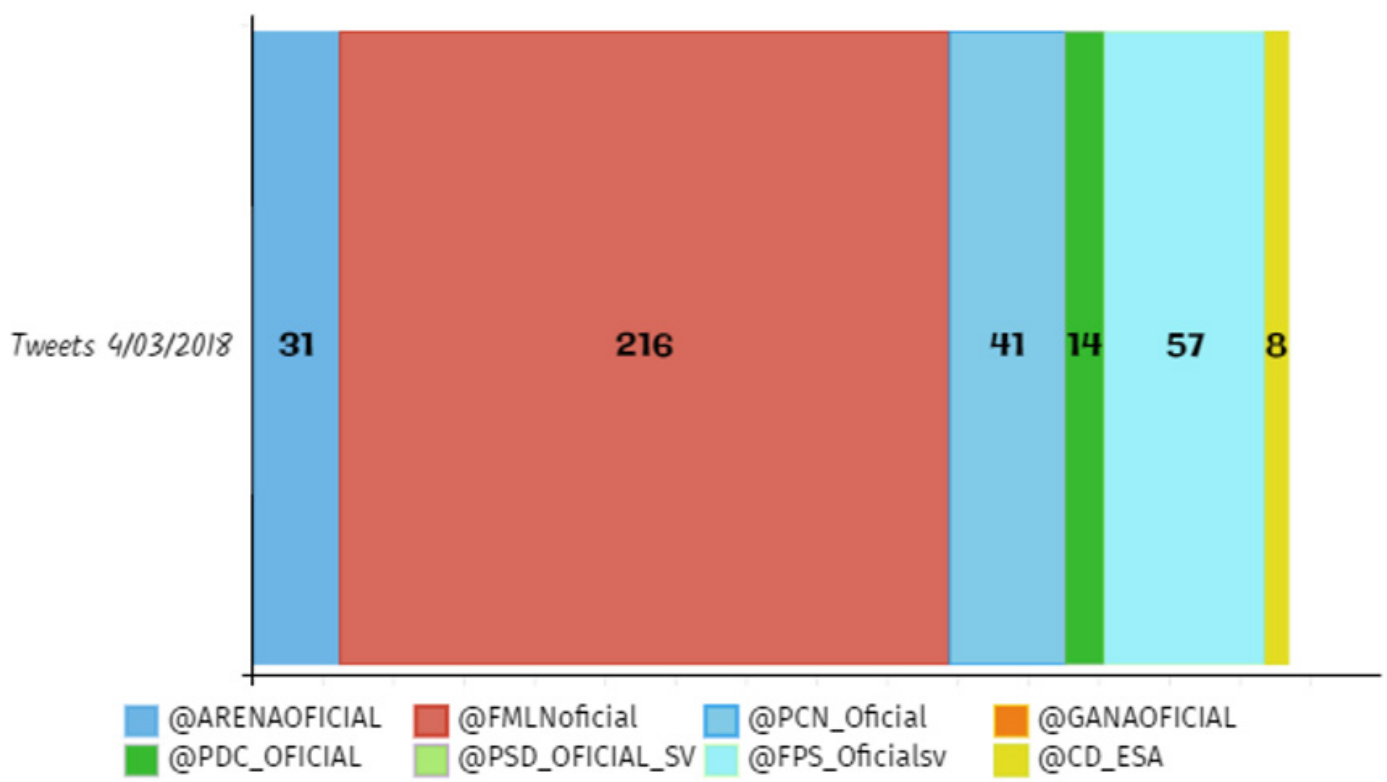

identificó que el perfil era actualizado utilizando la herramienta Botize, diseñada para automatizar tareas en Internet. Es decir, sus publicaciones fueron programadas y no cumplieron con un principio importante en la comunicación: la interacción efectiva. Ninguno de sus tweets hizo referencia al día de las elecciones, todos eran mensajes relativos a su plataforma legislativa y que además habían sido publicados de manera repetitiva en días anteriores.

\section{Los hashtags}

El hashtag se ha convertido actualmente en una herramienta indispensable para la creación de contenidos y el aumento de participación de los usuarios, así como para la difusión de información relevante sobre los productos, eventos, noticias, y tendencias de la industria o sector (Guerra, 2018). Un hashtag consta de palabras o frases, sin espacios, y que son precedidas de un signo almohadilla [\#]. Esto ha hecho posible que los usuarios puedan participar en conversaciones sobre un tema particular, y de esta manera quedar agrupadas bajo una misma etiqueta. Incluso la búsqueda de información es más efectiva, ya que al estar agrupados resulta más fácil la identificación en una búsqueda rápida en la web o en la misma red social.

El 4 de marzo de 2018 se utilizaron muchos hashtags entre los usuarios de Twitter; pero gracias a la herramienta de Trendsmap.com se pudo hacer una georreferenciación en tiempo 


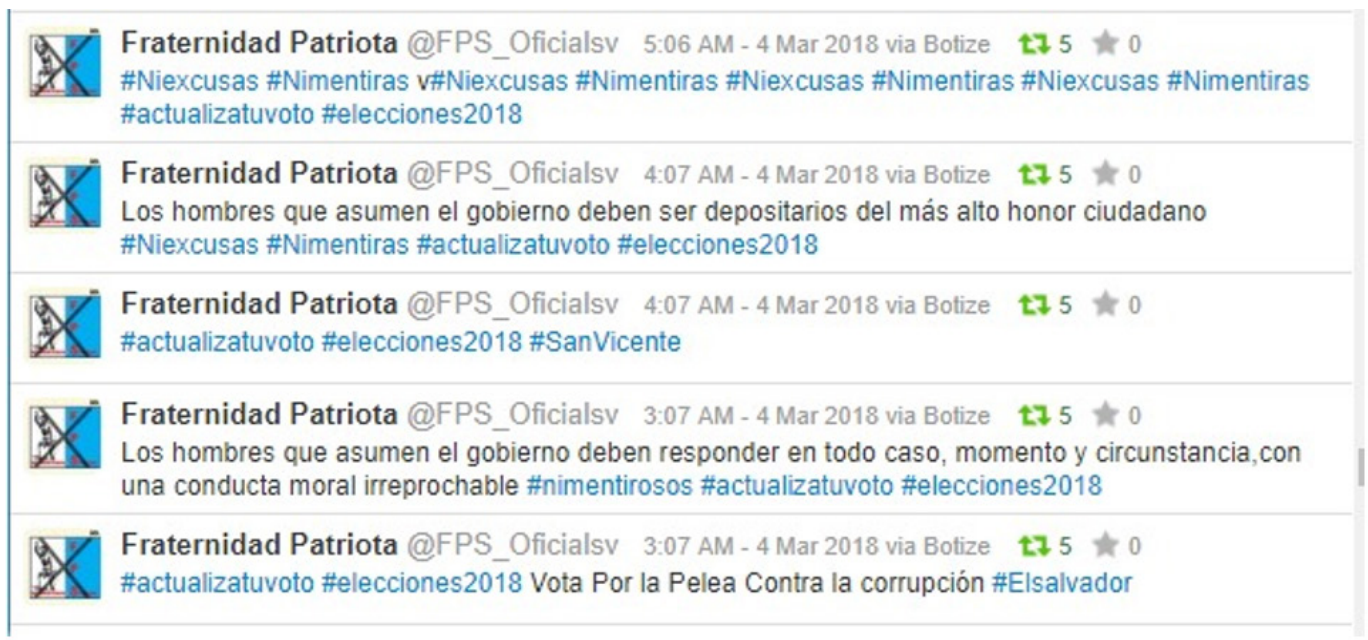

Figura n. ${ }^{\circ}$ 3. Imagen de referencia de los tweets publicados por el partido FPS. Fuente: perfil de Twitter@FPS_Oficialsv

real de aquellos hashtags que más se compartieron entre las ocho de la mañana y las diez de la noche. De hecho se pueden ubicar entre esas horas un total de 32 hashtags más sobresalientes en el país, entre los que destacan algunos de los usados por los medios de comunicación y la difusión de sus programas electorales, así como los usados por los partidos políticos. Por otro lado, también puede tenerse una idea de cómo funcionaron las conversaciones en Twitter durante las catorce horas que se monitoreó dicha red.

Por otra parte, de los 32 hashtags que se compartieron en el día de la elección de alcaldes y diputados 2018, hubo algunos que fueron permanentes; es decir que desde tempranas horas de la mañana hasta el final del día fueron usados por las audiencias de Twitter. Tal es el caso de:

- \#minutoaminuto (programa especial de Telecorporación Salvadoreña).

- \#votogmv (Grupo Megavisión).
- \#horadelaverdad33 (programa especial de Canal 33).

- \#eleccionessv (usado como referencia de información sobre las votaciones).

- \#elsalvador.

- \#votonulo2018 (campaña impulsada por varios movimientos con el fin de anular el voto).

- \#rumboalasurnasgmv (programa especial del Grupo Megavisión)

- \#elecciones2018.

- \#efelectoral (refiriéndose a información del periódico digital www.elfaro.net)

- \#votonulo (parte de la campaña de varios movimientos que impulsa el voto nulo.

En su mayoría, los hashtags utilizados estuvieron relacionados al día de las elecciones; pero también tomaron protagonismo los relacionados al fútbol y al partido del Barcelona contra el Atlético de Madrid, así como los vinculados a la entrega de los premios Oscar 2018. 


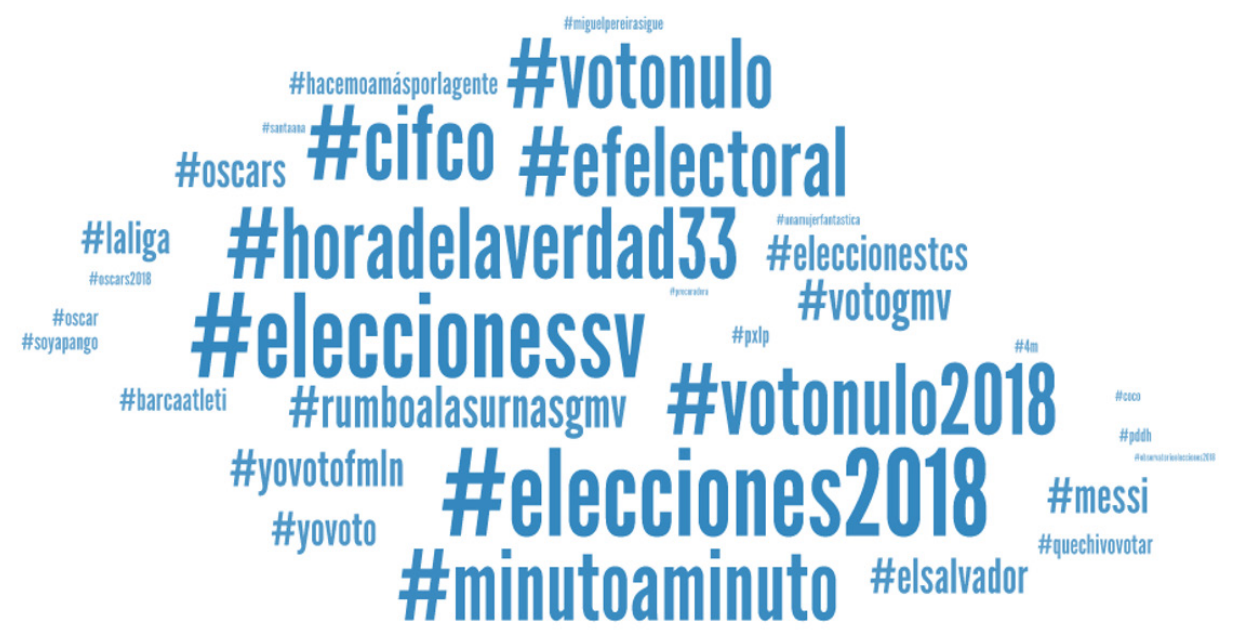

Figura $n .^{\circ} 4$. Hashtags compartidos en Twitter el día de la elección.

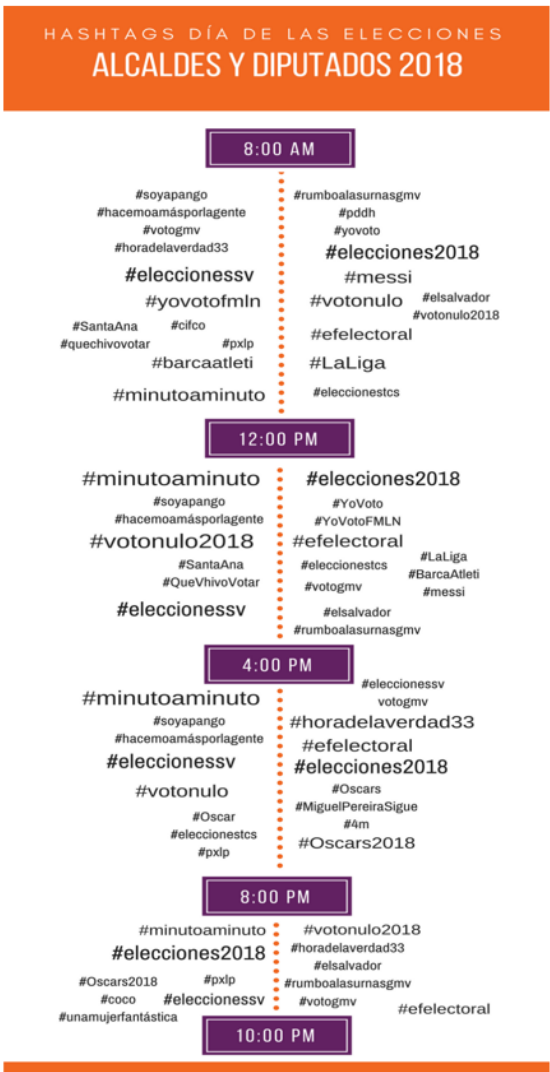

\section{Las palabras}

Para conocer los temas que marcaron la agenda de conversaciones en Twitter se utilizó la herramienta Trendsmap.com, a fin de monitorear las palabras más utilizadas por las audiencias; y tal como se hizo con los hashtags, las palabras se monitorearon, de manera georreferenciada, cada dos horas, entre las ocho de la mañana y las diez de la noche.

Estas palabras o temas son conocidos como trending topic; es decir, las palabras clave más utilizadas en un plazo de tiempo concreto en Twitter (Diccionario Inbound Marketing ,2018). Esta red de microblogging se ha trasformado, gracias a sus usuarios, en uno de los principales canales para advertir las tendencias en la web.

El día de las elecciones para diputaciones y concejos municipales 2018, en El Salvador, un total de 48 palabras estuvieron dominando las conversaciones, la mayoría sobre el tema electoral y una pequeña porción sobre el fútbol. 
A diferencia de los hashtags, la ceremonia número noventa de la entrega de los Premios Óscar 2018 no estuvo dominando la conversación nacional en Twitter. papeletas con imágenes de votos nulos; así como algunas palabras relacionadas a los resultados; por ejemplo, surgieron palabras como: debacle, culpables y cúpula, en donde las audiencias ya

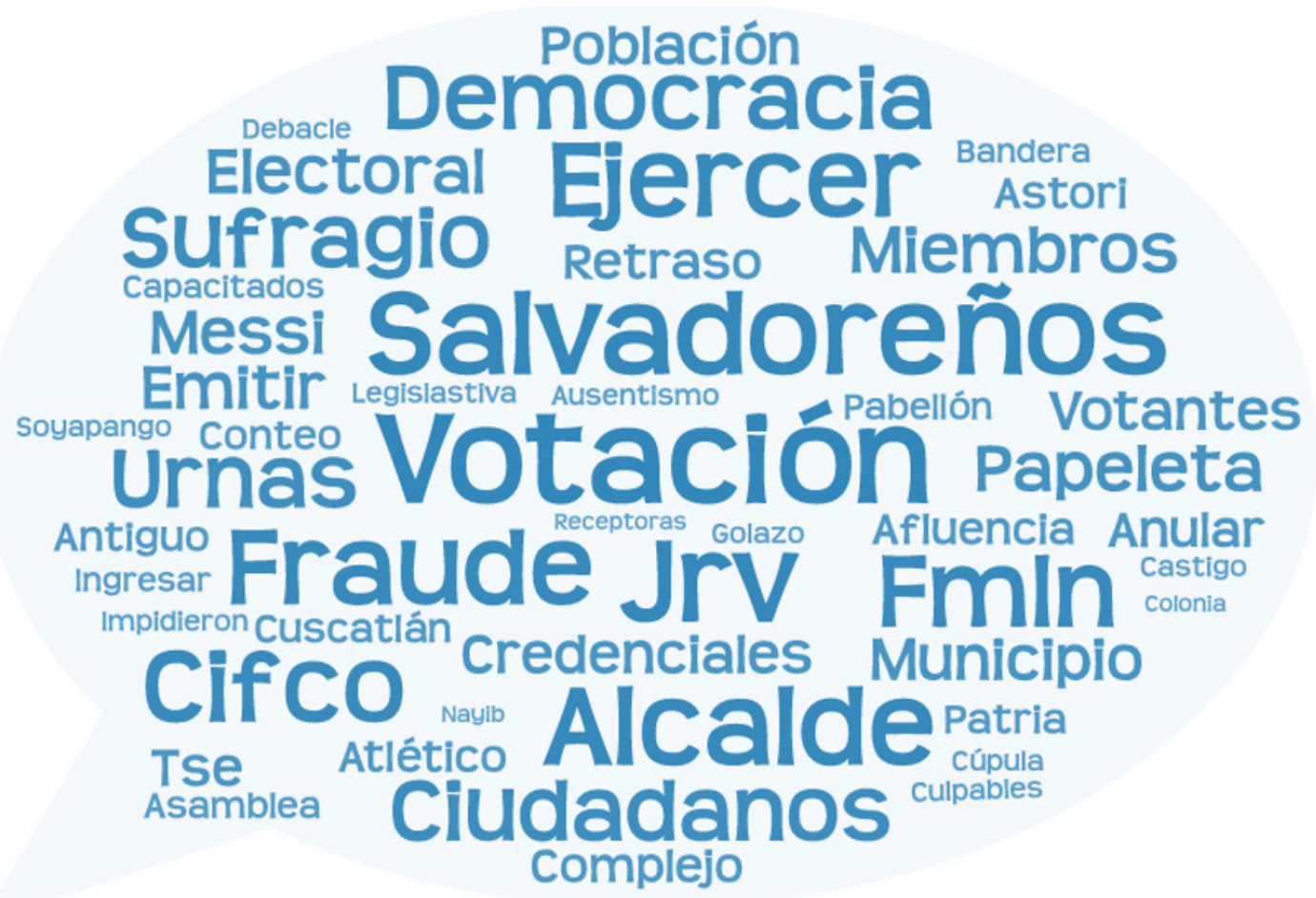

Figura $n .^{\circ}$ 5. Tendencia de palabras compartidas en Twitter.

Al analizar los temas, claramente puede advertirse cuáles fueron las conversaciones que predominaron en la red. Por ejemplo, por la mañana el retraso en el arranque de operaciones de algunos de Centros de Votación fue algo que captó la atención de los usuarios; así como el hecho de que despacharan de algunos Centros de Votación a miembros de las mesas (JRV) sin afiliación partidaria y que habían sido capacitados por el TSE. Por la tarde y noche, los temas más sobresalientes estuvieron relacionados con la anulación de voto, las daban posición con respecto a los bajos números de votación que obtuvo el partido oficial.

\section{Los usuarios}

El 4 de marzo de 2018 también se pudieron identificar los usuarios de Twitter que generaron más conversaciones en la red. Estos usuarios conocidos también como influencers, son aquellas personas que tienen una gran presencia y difunden contenidos de interés a través de las redes sociales como YouTube, 


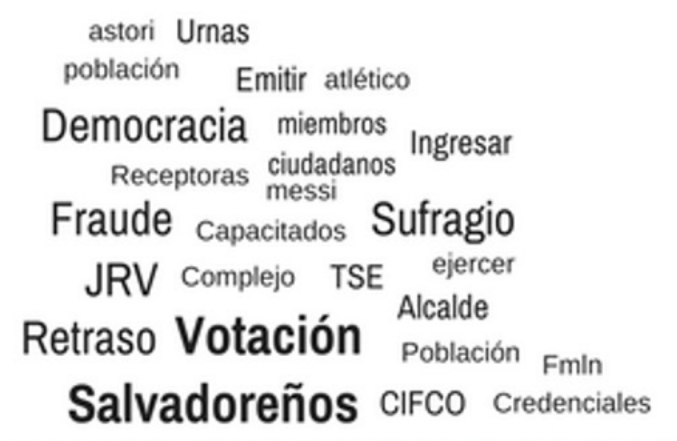

Votación messi bandera debacle

10:00 PM Anular Patria

Ausentismo

Alcalde

Salvadoreños

Conteo JRV Cifco ${ }^{\text {Nayib }}$

castigo afluencia Culpables

Democracia Cúpula

Figura n. ${ }^{\circ}$. Trending Topic en Twitter del día de la elección.

Twitter, Instagram, entre otros (Universidad Complutence de Madrid, 2018).

La presencia que dicho usuario tiene online es el capital de todo influencer. Sin embargo, el alcance por sí solo no lo es todo, porque lo que en realidad cuenta es el nivel de impacto social. En primera instancia cualquier persona o cuenta de Twitter puede ser un influencer: periodistas, blogueros, youtubers, celebridades, políticos, autores o profesionales, medios de comunicación, etc. La reputación y la credibilidad del infuencer también son factores importantes para que los usuarios generen interacción en la web.

Las cuentas más sobresalientes el 4 de marzo de 2018 fueron la del Tribunal Supremo Electoral(@ tseelsalvador), las de los candidatos a alcalde por San Salvador, Jackeline Rivera (@JackelineRA_) y Ernesto Muyshondt (@emuyshondt); la del alcalde de la capital Nayib Bukele (@ nayibbukele) y la del diputado presidente por la Asamblea Legislativa, Guillermo Gallegos (@ ggallegos24).

Es importante señalar que la cuenta del partido oficial (@FMLNoficial) que fue de las cuentas más activas durante la jornada electoral, disminuyó su actividad a partir de las seis de la tarde y solo publicó un tweet que contenía un comunicado. Por otra parte, las cuentas de los medios tradicionales de comunicación fueron las que tuvieron mayor actividad a lo largo del día.

\section{Conclusiones}

Este estudio pretende sumarse al debate que existe en Latinoamérica sobre la importancia 


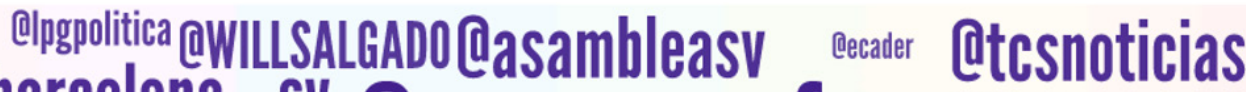 @sanchezceren @FMLNoficial @areEAdeficial @JackelineRA @palomo_espn

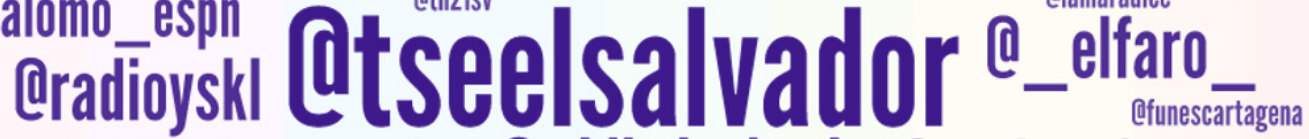 Qeemelulysts Qggallegos24 @elmundo_sv Qwaraujjo64}

Figura $n .^{\circ} 7$. Influencers del día de las elecciones para diputaciones y concejos municipales 2018.

y el uso de las redes sociales, y en específico de Twitter, en los procesos electorales. Para esto, plantea una discusión inicial sobre el alcance de la comunicación política y las redes sociales advirtiendo sobre el poco valor que los políticos salvadoreños le dan a una herramienta como Twitter. E1 nivel de presencia de los candidatos y partidos políticos fue pobre, y en el caso de los secretarios generales de los partidos se evidenció una participación casi nula.

Pese a la poca utilización de la herramienta, en el análisis de contenido realizado a las publicaciones o tweets se demuestra que fueron los mensajes hacia los usuarios y la difusión de sus actividades como visitas a medios de comunicación o actividades con seguidores y militantes las que más importancia tuvieron. También se puede evidenciar, por medio de la diversidad de dispositivos empleados para la emisión de tweets, que algunas de las cuentas de secretarios generales o de los candidatos no son gestionadas directamente por ellos, lo que provoca que la gestión de la comunicación sea cada vez más impersonal y que los usuarios la perciban como falsa.

No se puede hablar de un aprovechamiento de las potencialidades de interacción entre políticos, partidos y ciudadanos a través de Twitter el día de las elecciones ya que la interacción no estuvo presente en la red. Sólo dos candidatos interactuaron con sus seguidores; por lo demás, ni los secretarios generales ni los partidos políticos se relacionaron son sus seguidores, con lo que se demuestra que el aprovechamiento de dicha herramienta no es parte de las estrategias de comunicación digital de ninguno de los partidos políticos. 
En El Salvador no se ha producido una revolución en las estrategias de campaña a través del Internet o las redes sociales, de modo que por medio de ellos se fomente la interacción o el diálogo. De hecho, el parangón que se puede establecer entre las estrategias online y offline hace que solo pueda hablarse de un cambio de formato para la propaganda tradicional; en el sentido que tener una cuenta de Twitter parece ser solo un requisito y no una herramienta o canal de comunicación que podría ser importante en la campaña.

\section{Bibliografía}

Abela,J.A. (marzo de 2012). Centro de Estudios Andaluces. Recuperado en marzo de 2018, de http://public.centrodeestudiosandaluces.es/ pdfs/S200103.pdf

Diccionario Inbound Marketing. (marzo de 2018). Qué es trending topic. 40 de Fiebre. Disponible en: https:/www.40defiebre.com/ que-es/trending-topic-tt/
Echeverría, J. (1999). Telepolis y Tercer entorno. Barcelona: Destino.

Guerra, C. (marzo de 2018). Hashtag qué es y para qué sirve. Carlos Guerra Terol. Disponible en: https://carlosguerraterol.com/hashtag-quees-para-que-sirve-como-usar/

Mancera, A., \& Pano, A. (2013). El discurso politico de Twitter. Barcelona: Anthropos.

Tecnología hecha palabra. (22 de junio de 2017). Salud y Tecnología. Disponible en: http://www. tecnologiahechapalabra.com/salud/eventos/ articulo.asp? $\mathrm{i}=10916$

Universidad Complutence de Madrid. (marzo de 2018). El marketing de influencia y los influencers. Nticmaster.com Disponible en: https://www.nticmaster.com/ blogmastercom munitymanager/277-e1marketing-de-influencia-y-los-influencers.html 